\title{
Amentoflavone Enhances the Therapeutic Efficacy of Sorafenib by Inhibiting Anti-apoptotic Potential and Potentiating Apoptosis in Hepatocellular Carcinoma In Vivo
}

\author{
JAI-JEN TSAI ${ }^{1,2,3}$, FEI-TING HSU ${ }^{4,5,6}$, PO-JUNG PAN ${ }^{2,7,8}$, CHIA-WEN CHEN $^{9,10,11}$ and YU-CHENG KUO ${ }^{12,13}$ \\ ${ }^{1}$ Division of Gastroenterology, Department of Medicine, \\ National Yang-Ming University Hospital, I-Lan, Taiwan, R.O.C.; \\ ${ }^{2}$ Cancer Medical Care Center, National Yang Ming University Hospital, I-Lan, Taiwan, R.O.C.; \\ ${ }^{3}$ Department of Nursing, Cardinal Tien Junior College of Healthcare \& Management, I-Lan, Taiwan, R.O.C.; \\ ${ }^{4}$ Department of Radiology, School of Medicine, Taipei Medical University, Taipei, Taiwan, R.O.C.; \\ ${ }^{5}$ Department of Medical Imaging, Taipei Medical University Hospital, Taipei, Taiwan, R.O.C.; \\ ${ }^{6}$ Translational Imaging Research Center, Taipei Medical University Hospital, Taipei, Taiwan, R.O.C.; \\ ${ }^{7}$ Department of Physical Medicine and Rehabilitation, \\ National Yang-Ming University Hospital, I-Lan, Taiwan, R.O.C.; \\ ${ }^{8}$ Faculty of Medicine, School of Medicine, National Yang-Ming University, Taipei, Taiwan, R.O.C.; \\ ${ }^{9}$ Department of Anesthesiology, School of Medicine, China Medical University, Taichung, Taiwan, R.O.C.; \\ ${ }^{10}$ Department of Anesthesiology, China Medical University Hospital, Taichung, Taiwan, R.O.C.; \\ ${ }^{11}$ Department of Anesthesiology, Asia University Hospital, Taichung, Taiwan, R.O.C.; \\ ${ }^{12}$ Department of Radiation Oncology, China Medical University Hospital, \\ China Medical University, Taichung, Taiwan, R.O.C.; \\ ${ }^{13}$ Department of Radiation Oncology, Show Chwan Memorial Hospital, Changhua, Taiwan, R.O.C.
}

\begin{abstract}
Background/Aim: In a previous study, we showed that amentoflavone promotes sorafenib-induced apoptosis in hepatocellular carcinoma (HCC) cells in vitro. However, whether amentoflavone augments anticancer efficacy of sorafenib in HCC in vivo is unknown. The aim of the present study was to verify the anticancer effect of amentoflavone combined with sorafenib in HCC in vivo. Materials and Methods: HCC SK-Hepl tumor-bearing mice were treated with vehicle, sorafenib, amentoflavone, or combination for 14 days, respectively. Effect of sorafenib, amentoflavone, or their combination on tumor growth, anti-apoptotic potential, apoptotic signaling and general toxicity were evaluated with digital caliper, immunohistochemistry staining and body weight. Results: Our results demonstrated that amentoflavone significantly enhanced sorafenib-inhibited tumor growth and
\end{abstract}

Correspondence to: Dr. Yu-Cheng Kuo, Department of Radiation Oncology, China Medical University Hospital, China Medical University, Taichung, Taiwan, R.O.C. Tel: +886 422052121 \#7450, e-mail: shapico22@gmail.com

Key Words: Amentoflavone, sorafenib, hepatocellular carcinoma, ERK, AKT, apoptosis. expression of ERK/AKT phosphorylation and anti-apoptotic proteins compared to single-agent treatment. Additionally, amentoflavone also triggered sorafenib-induced apoptosis through extrinsic and intrinsic apoptotic pathways. Conclusion: Amentoflavone boosts therapeutic efficacy of sorafenib through blockage of anti-apoptotic potential and induction of apoptosis in HCC in vivo.

Hepatocellular carcinoma (HCC) is a prevalent cancer type and also the leading cause of cancer death among Taiwanese. Genomic alterations affecting matrix metalloproteinase-1 (MMP-1), tumor necrosis factor-alpha (TNF- $\alpha$ ), and DNA double-strand break repair gene XRCC7 genotypes result in increased HCC risk in Taiwanese males (1-3).

Sorafenib (Nexavar), an oral multi kinase inhibitor against Raf kinase and several receptor tyrosine kinases, has been approved for the treatment of advanced hepatocellular carcinoma (HCC) (4). Sorafenib blocks Raf kinase signaling transduction and inhibits activity of vascular endothelial growth factor receptor 2 (VEGFR2) and platelet-derived growth factor receptor (PDGFR) leading to inhibition of tumor growth and angiogenesis in HCC (5). Patients with advanced HCC have an unsatisfactory response to sorafenib treatment and resultant median overall survival is less than 


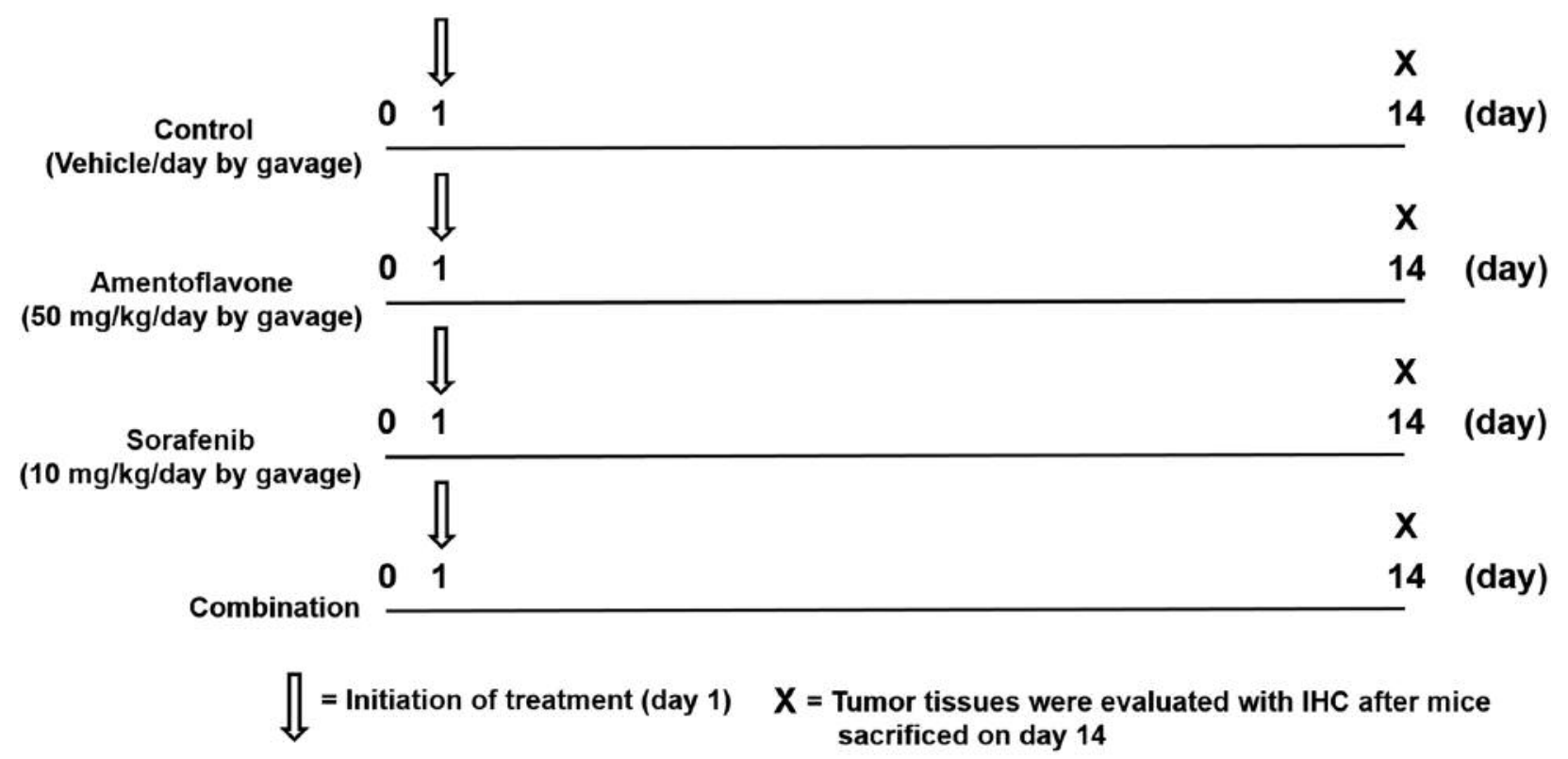

Figure 1. Flow chart of experimental protocol.

11 months (6). Lu et al. observed longer survival in patients receiving optimal dose of sorafenib in comparison to those receiving suboptimal dose (7). Yoshimoto et al. suggested the reasonable use of sorafenib before surgery in advanced HCC patients particularly those whose tumor responded to sorafenib (8). Therefore, development of sorafenib sensitizers which ameliorate efficacy of sorafenib against HCC is very important for advanced HCC patients under sorafenib treatment.

Flavonoids, extracted from natural products, Chinese herbal medicines, and phytochemicals, possess anticancer properties through blockage of multiple signal transduction pathways, initiation of apoptosis, and up-regulation of anticancer immune response (9-10). Flavonoid compounds such as wogonin (5,7 dihydroxy 8 methoxyflavone), fisetin, and quercetin are presented as potent sorafenib sensitizers to enhance anticancer activity of sorafenib in HCC, melanoma, and glioma, respectively (11-13). Flavonoids and sorafenib combination may be a potential strategy for treatment of HCC.

Amentoflavone, a flavonoid derived from scutellaria baicalensis, has been indicated to inhibit metastatic potential through suppression of NF-kB activation in breast cancer and osteosarcoma cells (14-15). In a previous study, we found that amentoflavone promotes sorafenib-induced apoptosis through extrinsic and intrinsic pathways in hepatocellular carcinoma in vitro (16). However, whether amentoflavone increases therapeutic efficacy of sorafenib in HCC in vivo is ambiguous. The aim of the present study was to verify the therapeutic efficacy of amentoflavone combined with sorafenib in SK-Hep1 HCC-bearing mice. Here, we demonstrated amentoflavone as a sorafenib sensitizer which enhances therapeutic effi-cacy of sorafenib through suppression of anti-apoptotic potential and induction of apoptosis in HCC in vivo.

\section{Materials and Methods}

Chemicals and agents. Amentoflavone and DMSO were purchased from Sigma-Aldrich (St. Louis, MO, USA). Sorafenib (Nexavar) was kindly provided from Bayer Health Care Pharmaceuticals, Inc. (Whippany, NJ, USA). Culture related products, including Dulbecco's modified Eagle's medium (DMEM), fetal bovine serum (FBS), Lglutamine and penicillin-streptomycin (PS) were obtained from Gibco/Life Technologies (Waltham, MA, USA). Immunoperoxidase secondary detection kit was bought from Millipore (Billeri-ca, MA, USA). Matrigel matrix was purchased from Corning Incorporated (Corning, NY, USA).

Cell culture. Human hepatocellular carcinoma SK-Hep1 cells were provided by professor Jing-Gung Chung at Department of Biological Science and Technology, China Medical University, (Taichung, Taiwan). Cells were cultured with DMEM containing $10 \%$ FBS, $2 \mathrm{mM}$ L-glutamine, and PS $(100 \mathrm{U} / \mathrm{ml}$ and $100 \mu \mathrm{g} / \mathrm{ml})$ and incubated at $37^{\circ} \mathrm{C}$ under a humidified atmosphere of $95 \%$ air and $5 \% \mathrm{CO}_{2}(17)$.

Establishment of tumor-bearing animal model. Six-week-old nude mice were brought from the National Laboratory Animal Center, Taipei, Taiwan. $1 \times 10^{7}$ SK-Hep1 cells were suspended in $150 \mu \mathrm{l}$ mix-ture of serum-free DMEM and matrigel (2:1) and inoculated subcutaneously in the right legs of nude mice (18). 

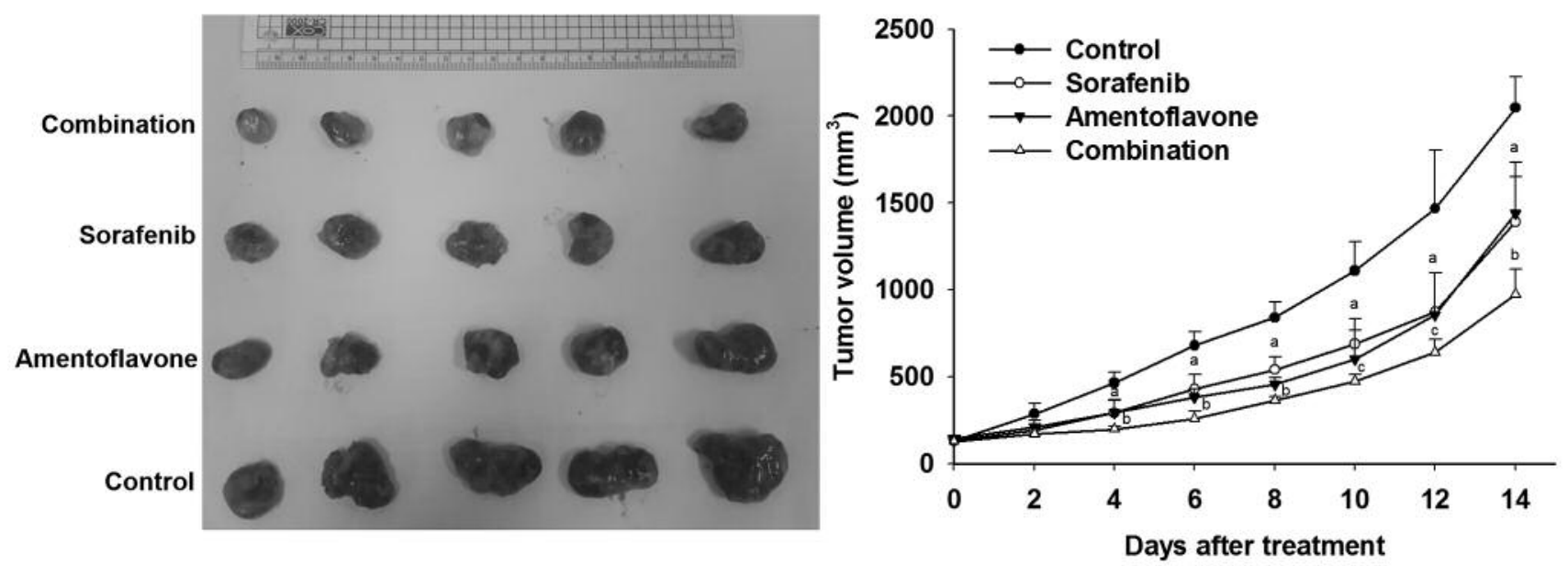

Figure 2. Effect of sorafenib, amentoflavone, and their combination on tumor growth in SK-Hep1 HCC bearing mice. Mice were treated with vehicle (100 $\mu \mathrm{l} \mathrm{PBS}$ plus $50 \mu \mathrm{l}$ DMSO per day), sorafenib (10 mg/kg/day), amentoflavone (50 mg/kg/day), or their combination (sorafenib $10 \mathrm{mg} / \mathrm{kg} \mathrm{plus}$ amentoflavone $50 \mathrm{mg} / \mathrm{kg}$ per day) by gavage for 14 days, respectively. Tumor growth was measured by digital caliper and tumor volume was calculated using the formula $0.523 \times$ length $\times$ width $\times$ thickness. ${ }^{a} p<0.01$ compared to vehicle group. ${ }^{b} p<0.01$ and ${ }^{c} p<0.05$ compared to sorafenib or amentoflavone group.

Experimental protocol (Figure 1). When average tumor volume reached about $130 \mathrm{~mm}^{3}$, mice were randomized into four groups ( $\mathrm{n}=5 /$ group), vehicle group [treated with $100 \mu \mathrm{l}$ phosphate-buffered solu-tion (PBS) plus $50 \mu$ dimethyl sulfoxide $(0.1 \%$ DMSO) by gavage daily for 14 days] and sorafenib group (treated with $10 \mathrm{mg} / \mathrm{kg} /$ day by gavage for 14 days), amentoflavone group (treated with $50 \mathrm{mg} / \mathrm{kg}$ /day by gavage for 14 days), and combination group (treated with $10 \mathrm{mg}$ sorafenib plus $50 \mathrm{mg}$ amentofla-vone/ $\mathrm{kg} /$ day by gavage for 14 days). Initiation of treatment on day 1 , tumor growth and body weights of mice were measured on day $0,2,4,6,8,10,12,14$ after treatment. Mice were sacrificed on day 14 for IHC staining of tumor tissues from each group. All experiments were repeated at least three times and complied with the guidance of institutional animal care (IACUC number approval by Taipei Medical University: LAC-2016-0028).

Immunohistochemistry (IHC) staining. Mice were sacrificed on day 14 after treatment and tumors were fixed with $4 \%$ paraformaldehyde (PFA) at $4^{\circ} \mathrm{C}$. Paraffin-embedded tumor tissue section with $5 \mu \mathrm{m}$ thickness was performed by Bio-Check Laboratories Ltd. (New Taipei City, Taiwan). According to the instructions provided with the kit, IHC staining was used to determine protein levels in tumor tis-sues. The sections were immunohistochemically stained with MCL-1 (BioVision, Milpitas, CA, USA), AKT (Ser473), C-FLIP, active caspase-3 (Cell Signaling Technology, Inc., Danvers, MA, USA), XIAP, active caspase-8, and -9 (Thermo Fisher Scientific, Fremont, CA, USA) antibodies, respectively. The stained sections were scanned at $100 \mathrm{X}$ magnification by using the microscopy-based TissueFAXS plat-form (TissueGnostics, Vienna, Austria). Positive expression of IHC indices in tumor tissues was quantified with ImageJ software version 1.50 (National Institutes of Health, Bethesda, MD, USA).

Statistical analysis. The difference between two groups (treatment and control group) was compared by Student's $t$-test. Results are showed as means \pm standard error. The $p$-value smaller than 0.05 was de-fined as statistically significant difference.

\section{Results}

Amentoflavone enhances sorafenib-inhibited tumor growth in SK-Hepl tumor-bearing mice. Effect of amentoflavone, sorafenib, or combination treatment on tumor growth in SK-Hep1 HCC bearing mice was evaluated with digital caliper. All the treatment groups significantly reduced tumor growth as compared to that of control group. Tumor volumes of the sorafenib group were similar to that of amentoflavone group. Combination group had significantly smaller tumor volumes as compared to sorafenib or amentoflavone group on day 4-14 (Figure 2).

Amentoflavone promotes sorafenib-inhibited anti-apoptotic potential and triggers sorafenib-induced apoptosis in SKHepl tumor bearing mice. All the treatment groups significantly decreased expression of anti-apoptotic proteins (MCL-1, C-FLIP, and XIAP) as compared to that of control group. Combination group had significantly lower protein levels of MCL-1, C-FLIP, and XIAP as compared to that of sorafenib group or amentoflavone group. Notably, the protein levels of AKT (Ser473) was not changed by sorafenib treatment. Both amentoflavone and combination group significantly inhibited protein levels of AKT (Ser473) by 57 and $56 \%$ compared to that of control, respectively (Figure $3 \mathrm{~A})$. All treatment groups significantly triggered activation of caspase- $9,-8$, and -3 compared to that of the control 
A
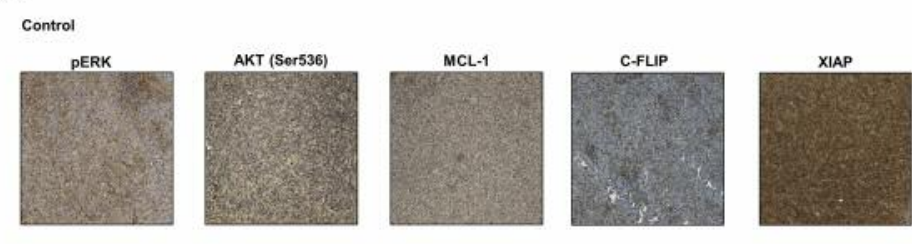

Sorafenib
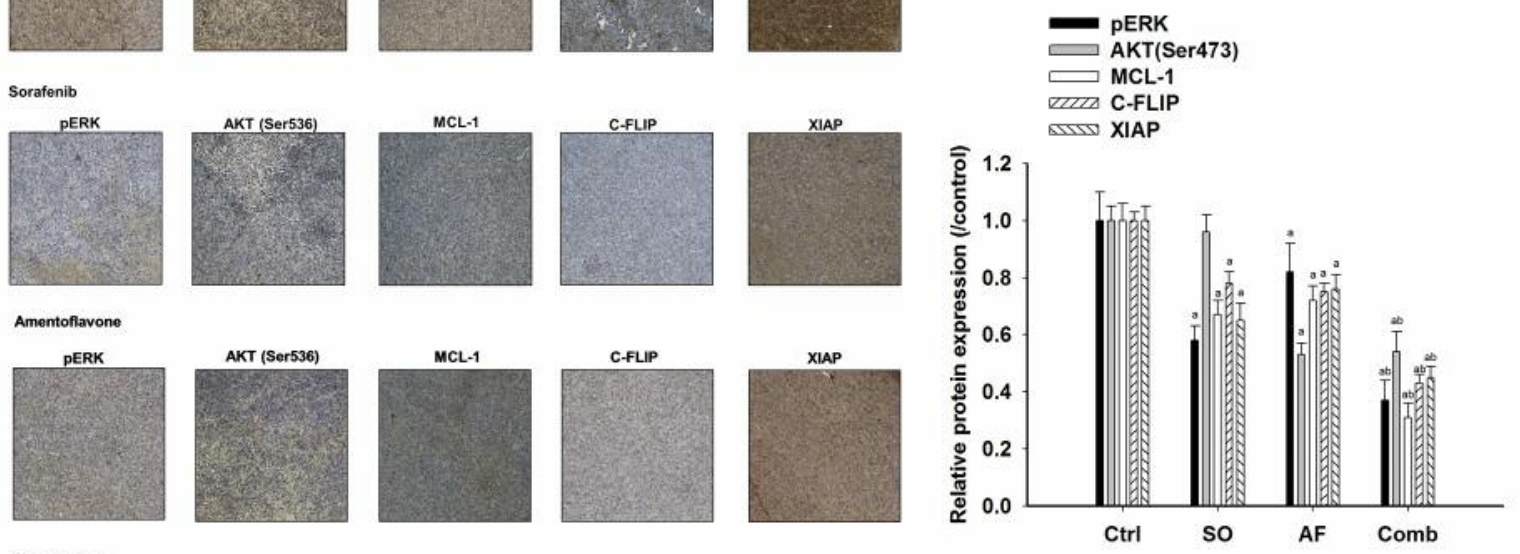

Combination
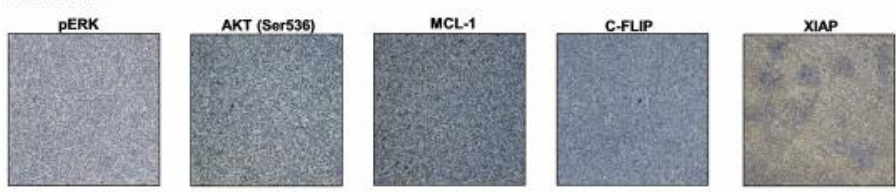

B
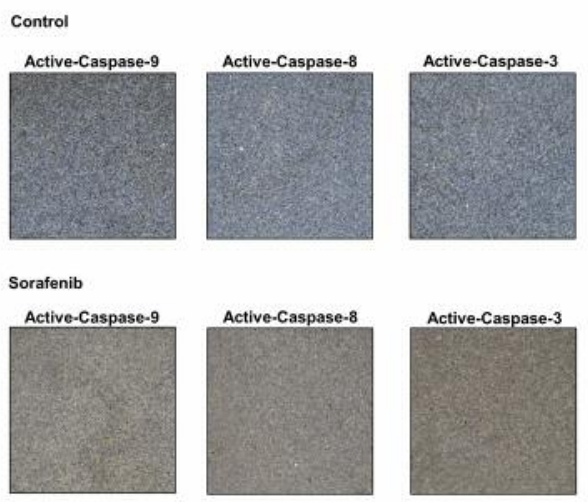

Amentoflavone
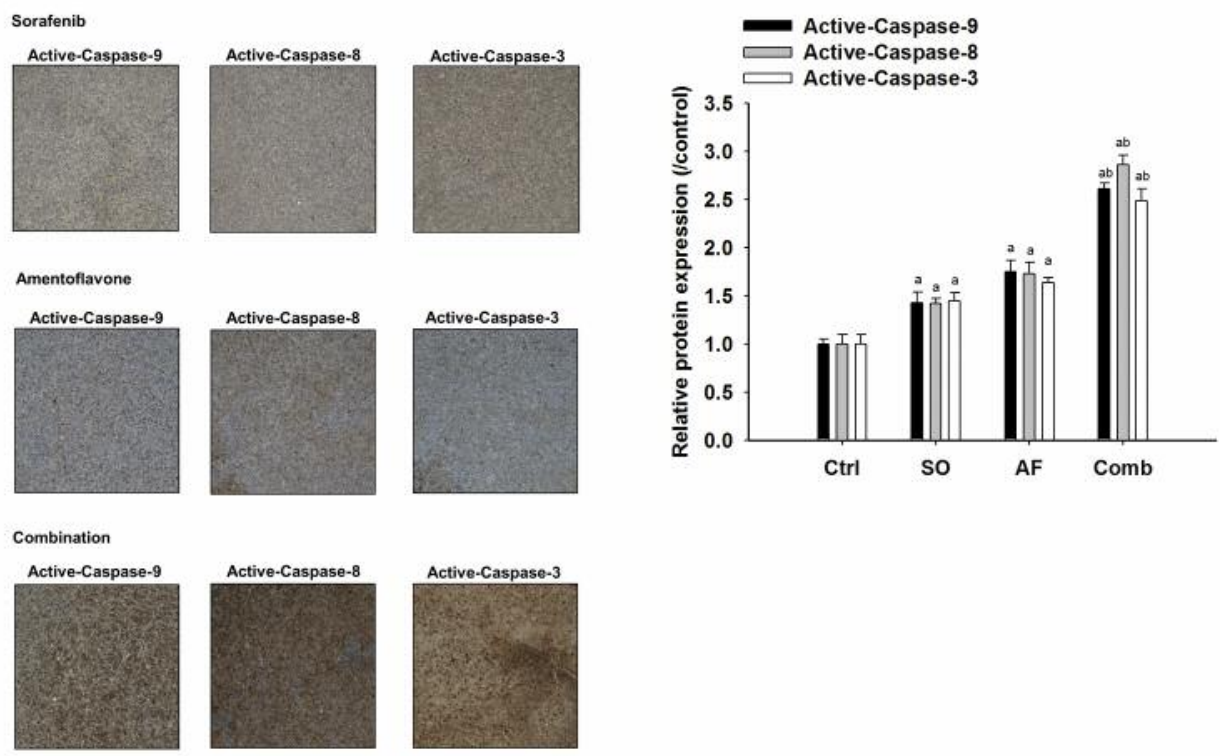

Figure 3. Effect of sorafenib, amentoflavone, or their combination on anti-apoptotic potential and apoptosis signaling in SK-Hep1 tumor-bearing mice. Mice were sacrificed on day 14 after treatments and protein expression of HCC tissues were investigated with IHC staining. A) Protein levels of pERK, AKT (Ser473), Mcl-1, C-FLIP, and XAIP. B) Protein expression of active-capase-9, -8, and -3. ap $<0.01$ compared to vehicle group and $b_{p}<0.01$ compared to sorafenib or amentoflavone group. 


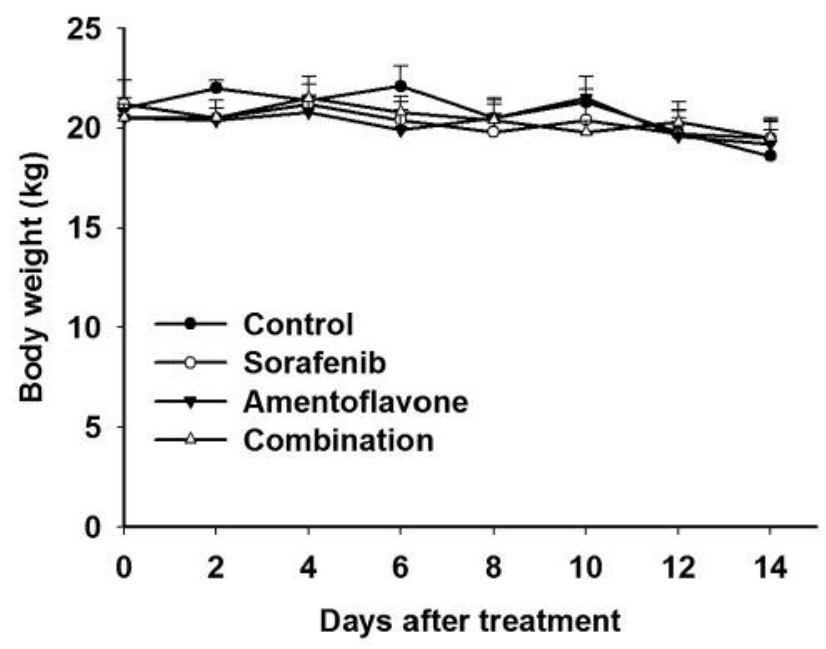

Figure 4. Effect of sorafenib, amentoflavone, or combination on body weight in SK-Hepl HCC bearing mice. Toxicity of treatment was monitored with body weight.

group. Combination group had significantly higher activation of caspase- $9,-8$, and -3 as compared to that of sorafenib or amentoflavone group (Figure 3B).

Toxicity analysis of treatments in SK-Hepl tumor bearing mice. We used body weight measurement to evaluate the general toxicity of treatments in SK-Hep1 HCC bearing mice. Figure 4 presented body weights that were not significantly changed by sorafenib, amentoflavone, or combination treatment in SK-Hep1 HCC bearing mice.

\section{Discussion}

Both rapidly accelerated fibrosarcoma (RAF)/mitogenactivated protein kinase kinase $(\mathrm{MEK}) / \mathrm{ERK}$ and phosphoinositide 3-kinase (PI3K)/AKT signaling pathways contribute to tumor growth, metastasis, angiogenesis, and anti-apoptotic potential in HCC (19). Sorafenib blocks $\mathrm{RAF} / \mathrm{MEK} / \mathrm{ERK}$ signaling transduction, but does not affect (PI3K)/AKT signaling pathway in HCC (5). Akt phosphorylation modulates acquired resistance to sorafenib and correlates to poor prognosis in HCC (20-21). Inhibition of AKT phosphorylation has been indicated to sensitize HCC to sorafenib. PI-103, a potent inhibitor of AKT (Ser473) phosphorylation, combined with sorafenib significantly suppressed epidermal growth factor (EGF)stimulated HCC cell proliferation by inhibiting both Raf/MEK/ERK and PI3K/AKT signaling transduction (22). Combination of PI-103 and sorafenib significantly inhibited tumor growth as compared to single-agent treatment in HCC in vivo (23). We demonstrated that combination of amentoflavone and sorafenib significantly reduces tumor growth, protein levels of pERK and AKT (Ser473) in HCC in vivo and ex vivo.

Anti-apoptotic proteins such as $\mathrm{X}$ linked inhibitor of apoptosis protein (XIAP), cellular FADD-like IL-1 $\beta$ converting enzyme (FLICE)-inhibitory protein (C-FLIP), and myeloid cell leukemia 1 are overexpressed in $\mathrm{HCC}$ and linked to poor survival of HCC patients. C-FLIP, MCL-1, and XIAP diminish anticancer agents-induced apoptosis through inhibition of extrinsic, intrinsic apoptosis, and caspase-3 activation, respectively (24). Lee et al., performed phase II study of AEG35156, antisense oligonucleotide to XIAP, combined with sorafenib in patients with advanced HCC. The combination of AEG35156 and sorafenib has additional activity in terms of objective response rates (ORR) and moderate benefit on progression-free survival (PFS) as compared to sorafenib alone (25). Hsu et al. presented overexpression of cyclin E1 and myeloid cell leukemia 1 ( $\mathrm{Mcl} 1$ ) reduces sorafenib-triggered apoptosis, whereas inhibition of cyclin E1 and $\mathrm{Mcl} 1$ promotes induction of apoptosis in HCC (26). We showed that amentoflavone significantly enhances sorafenib-inhibited expression of anti-apoptotic proteins MCL-1, C-FLIP, and XIAP in HCC ex vivo.

Many flavonoids augment sorafenib-inhibited cell growth by inducing apoptosis in cancers. Anti-cancer agents activate apoptosis signaling through initiation of extrinsic and intrinsic pathways (16). Wogonin, a flavonoid extracted from Scutellaria baicalensis, combined with sorafenib effectively kills HCC cells through apoptosis potentiation (11). Quercetin, a natural flavonoid derived from fruits and vegetables, enhances sorafenib-induced apoptosis through induction of intrinsic apoptotic pathway in human gliomas. Combination of quercetin and sorafenib significantly increase expression of active-caspase-3 and -9 (13). Fisetin, a dietary flavonoid existed in many fruits and vegetables, has been shown to potentiate sorafenib-induced apoptosis via activation of death receptor-5 mediated caspase-8/caspase- 3 and the mitochondria-dependent apoptotic pathway in cervical cancer HeLa cells (27). We demonstrated the combination of amentoflavone and sorafenib significantly trigger expression of activecaspase- $9,-8$, and -3 as compared to single-agent treatment in $\mathrm{HCC}$ ex vivo.

In conclusion, this study revealed amentoflavone as a sorafenib sensitizer to enhance sorafenib-inhibited tumor growth through inhibition of the anti-apoptotic potential and induction of extrinsic and intrinsic apoptotic pathways in HCC in vivo. We suggest that the combination of amentoflavone and sorafenib may be a potential strategy for treatment of patients with HCC.

\section{Conflicts of Interest}

The Authors declare no competing financial interests. 


\section{Acknowledgements}

This study was supported by Grants RD2017-021 and RD106039 from the National Yang-Ming University Hospital, I-Lan, Taiwan and Show Chwan Memorial Hospital, Taiwan. The Authors acknowledge the technical services provided by Clinical Medicine Research Laboratory of National Yang-Ming University Hospital, ILan, Taiwan and Translational Laboratory, Department of Medical Research, Taipei Medical University Hospital, Taipei, Taiwan.

\section{References}

1 Lai YL, Gong CL, Fu CK, Yueh TC, Tsai CW, Chang WS, Hsiao CL, Yen ST, Li HT, Jeng LB, Wang SC, and Bau DT: The contribution of matrix metalloproteinase-1 genotypes to hepatocellular carcinoma susceptibility in Taiwan. Cancer Genomics Proteomics 14: 119-125, 2017.

2 Yang MD, Hsu CM, Chang WS3, Yueh TC, Lai YL, Chuang CL, Wang SC, Jeng LB, Ji HX, Hsiao CL, Wu CN, Tsai CW, Chung $\mathrm{JG}$, and Bau DT: Tumor necrosis factor- $\alpha$ genotypes are associated with hepatocellular carcinoma risk in Taiwanese males, smokers and alcohol drinkers. Anticancer Res 35: 5417-5423, 2015.

3 Hsieh YH, Chang WS, Tsai CW, Tsai JP, Hsu CM, Jeng LB, and Bau DT: DNA double-strand break repair gene XRCC7 genotypes were associated with hepatocellular carcinoma risk in Taiwanese males and alcohol drinkers. Tumour Biol 36: 41014106, 2015.

4 Chiang IT, Liu YC, Wang WH, Hsu FT, Chen HW, Lin WJ, Chang WY and Hwang JJ: Sorafenib inhibits TPA-induced MMP-9 and VEGF expression via suppression of ERK/NF-kB pathway in hepatocellular carcinoma cells. In Vivo 26: 671-681, 2012.

5 Liu L, Cao Y, Chen C, Zhang X, McNabola A, Wilkie D, Wilhelm S, Lynch $\mathrm{M}$ and Carter C: Sorafenib blocks the RAF/MEK/ERK pathway, inhibits tumor angiogenesis, and induces tumor cell apoptosis in hepatocellular carcinoma model PLC/PRF/5. Cancer Res 66: 11851-11858, 2006.

6 Llovet JM, Ricci S, Mazzaferro V, Hilgard P, Gane E, Blanc JF, de Oliveira AC, Santoro A, Raoul JL, Forner A, Schwartz M, Porta C, Zeuzem S, Bolondi L, Greten TF, Galle PR, Seitz JF, Borbath I, Häussinger D, Giannaris T, Shan M, Moscovici M, Voliotis D, Bruix $\mathbf{J}$ and SHARP Investigators Study Group: Sorafenib in advanced hepatocellular carcinoma. N Engl J Med 359: 378-390, 2008.

7 Lu LC, Chen PJ, Yeh YC, Hsu CH, Chen HM, Lai MS, Shao YY, and Cheng AL: Prescription patterns of sorafenib and outcomes of patients with advanced hepatocellular carcinoma: a national population study. Anticancer Res 37: 2593-2599, 2017.

8 Yoshimoto T, Imura S, Morine Y, Ikemoto T, Arakawa Y, Iwahashi S, Saito YU, Takasu C, Ishikawa D, Teraoku H, Bando $\mathrm{Y}$, and Shimada M: The outcome of sorafenib therapy on unresectable hepatocellular carcinoma: experience of conversion and salvage hepatectomy. Anticancer Res 38: 501-507, 2018.

9 Liao CY, Lee CC, Tsai CC, Hsueh CW, Wang CC, Chen IH, Tsai MK, Liu MY, Hsieh AT, Su KJ, Wu HM, Huang SC, Wang YC, Wang CY, Huang SF, Yeh YC, Ben RJ, Chien ST, Hsu CW and Kuo WH: Novel investigations of flavonoids as chemopreventive agents for hepatocellular carcinoma. Biomed Res Int 2015: 840542, 2015.
10 Chiang IT, Wang WS, Liu HC, Yang ST, Tang NY and Chung JG: Curcumin alters gene expression-associated DNA damage, cell cycle, cell survival and cell migration and invasion in NCI-H460 human lung cancer cells in vitro. Oncol Rep 34: 1853-1874, 2015.

11 Rong LW, Wang RX, Zheng XL, Feng XQ, Zhang L, Zhang L, Lin Y, Li ZP and Wang X: Combination of wogonin and sorafenib effectively kills human hepatocellular carcinoma cells through apoptosis potentiation and autophagy inhibition. Oncol Lett 13: 5028-5034, 2017.

12 Pal HC, Baxter RD, Hunt KM, Agarwal J, Elmets CA, Athar M and Afaq F: Fisetin, a phytochemical, potentiates sorafenibinduced apoptosis and abrogates tumor growth in athymic nude mice implanted with BRAF-mutated melanoma cells. Oncotarget 6: 28296-28311, 2015.

13 Jakubowicz-Gil J, Langner E, Bądziul D, Wertel I and Rzeski W: Quercetin and sorafenib as a novel and effective couple in programmed cell death induction in human gliomas. Neurotox Res 26: 64-77, 2014.

14 Chen JH, Chen WL and Liu YC: Amentoflavone induces antiangiogenic and anti-metastatic effects through suppression of NF-kB activation in MCF-7 cells. Anticancer Res 35: 66856693, 2014

15 Pan PJ Tsai JJ and Liu YC: Amentoflavone inhibits metastatic potential through suppression of ERK/NF-kB activation in osteosarcoma U2OS cells. Anticancer Res 37: 4911-4918, 2017.

16 Chen WL, Hsieh CL, Chen JH, Huang CS, Chen WT, Kuo YC, Chen CY and Hsu FT: Amentoflavone enhances sorafenibinduced apoptosis through extrinsic and intrinsic pathways in sorafenib-resistant hepatocellular carcinoma SK-Hep1 cells in vitro. Oncol Lett 14: 3229-3234, 2017.

17 Liu YC, Wu RH and Wang WS: Regorafenib diminishes the expression and secretion of angiogenesis and metastasis associated proteins and inhibits cell invasion via NF-kB inactivation in SK-Hep1 cells. Oncol Lett 14: 461-467, 2017.

18 Della Peruta M, Badar A, Rosales C, Chokshi S, Kia A, Nathwani D, Galante E, Yan R, Arstad E, Davidoff AM, Williams R, Lythgoe MF and Nathwani AC: Preferential targeting of disseminated liver tumors using a recombinant adeno-associated viral vector. Hum Gene Ther 26: 94-103, 2015.

19 Steelman LS, Chappell WH, Abrams SL, Kempf RC, Long J, Laidler P, Mijatovic S, Maksimovic-Ivanic D, Stivala F, Mazzarino MC, Donia M, Fagone P, Malaponte G, Nicoletti F, Libra M, Milella M, Tafuri A, Bonati A, Bäsecke J, Cocco L, Evangelisti C, Martelli AM, Montalto G, Cervello M and McCubrey JA: Roles of the Raf/MEK/ERK and PI3K/PTEN/Akt/mTOR pathways in controlling growth and sensitivity to therapy-implications for cancer and aging. Aging (Albany NY) 3: 192-222, 2011.

20 Zhai B, Hu F, Jiang X, Xu J, Zhao D, Liu B, Pan S, Dong X, Tan G, Wei Z, Qiao H, Jiang H and Sun X: Inhibition of Akt reverses the acquired resistance to sorafenib by switching protective autophagy to autophagic cell death in hepatocellular carcinoma. Mol Cancer Ther 13: 1589-1598, 2014.

21 Nakanishi K, Sakamoto M, Yamasaki S, Todo S and Hirohashi S: Akt phosphorylation is a risk factor for early disease recurrence and poor prognosis in hepatocellular carcinoma. Cancer 103: 307-312, 2005.

22 Gedaly R, Angulo P, Hundley J, Daily MF, Chen C, Koch A and Evers BM: PI-103 and sorafenib inhibit hepatocellular carcinoma cell proliferation by blocking Ras/Raf/MAPK and PI3K/ AKT/mTOR pathways. Anticancer Res 30: 4951-4958, 2010. 
23 Gedaly R, Angulo P, Chen C, Creasy KT, Spear BT, Hundley J, Daily MF, Shah M and Evers BM: The role of PI3K/mTOR inhibition in combination with sorafenib in hepatocellular carcinoma treatment. Anticancer Res 32: 2531-2536, 2012.

24 Chiang IT, Chen WT, Tseng CW, Chen YC, Kuo YC, Chen BJ, Weng MC, Lin HJ and Wang WS: Hyperforin inhibits cell growth by inducing intrinsic and extrinsic apoptotic pathways in hepatocellular carcinoma cells. Anticancer Res 37: 161-167, 2017.

25 Lee FA, Zee BC, Cheung FY, Kwong P, Chiang CL, Leung KC, Siu SW, Lee C, Lai M, Kwok C, Chong M, Jolivet J and Tung $\mathrm{S}$ : Randomized phase II study of the X-linked inhibitor of apoptosis (XIAP) antisense AEG35156 in combination with sorafenib in patients with advanced hepatocellular carcinoma (HCC). Am J Clin Oncol 39: 609-613, 2016.
26 Hsu C, Lin LI, Cheng YC, Feng ZR, Shao YY, Cheng AL and Ou DL: Cyclin E1 inhibition can overcome sorafenib resistance in hepatocellular carcinoma cells through Mcl-1 suppression. Clin Cancer Res 22: 2555-2564, 2016.

27 Lin MT, Lin CL, Lin TY, Cheng CW, Yang SF, Lin CL, Wu CC, Hsieh YH and Tsai JP: Synergistic effect of fisetin combined with sorafenib in human cervical cancer HeLa cells through activation of death receptor-5 mediated caspase-8/caspase- 3 and the mitochondria-dependent apoptotic pathway. Tumour Biol 37: 6987-6996, 2016.

Received December 29, 2017

Revised February 14, 2018

Accepted February 15, 2018 